\title{
Ações de enfermagem para prevenção e tratamento de complicações em estomias intestinais
}

\author{
Nursing actions for prevention and treatment of complications in intestinal stomies \\ Acciones de enfermería para prevención y tratamiento de complicaciones en \\ estomias intestinales
}

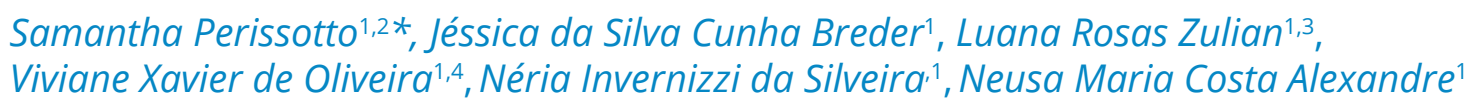

ORCID IDs

Perissotto S (iD https://orcid.org/0000-0003-2923-361X

Breder JSC (D) https://orcid.org/0000-0002-7424-5233

Zulian LR (iD https://orcid.org/0000-0001-9390-2502

Oliveira VX (D) https://orcid.org/0000-0002-0299-100X

Silveira NI (D) https://orcid.org/0000-0003-1677-4206

Alexandre NMC (D) https://orcid.org/0000-0001-5005-3360

\section{COMO CITAR}

Perissotto S, Breder JSC, Zulian LR, Oliveira VX de, Silveira NI da, Alexandre NMC. Ações de enfermagem para prevenção e tratamento de complicações em estomias intestinais: revisão integrativa. ESTIMA, Braz. J. Enterostomal Ther., 17: e0519. https://doi.org/10.30886/ estima.v17.638 PT

\section{RESUMO}

Objetivo: Identificar na literatura as principais ações de enfermagem para prevenção e tratamento das complicações mais frequentes nas estomias de eliminação intestinal. Método: Revisão integrativa que seguiu as fases: identificação do tema e seleção da questão de pesquisa; estabelecimento de critérios para inclusão e exclusão de estudos; categorização dos estudos; avaliação dos estudos incluídos na revisão integrativa; interpretação dos resultados; e síntese do conhecimento. O período utilizado foi de janeiro de 2007 a junho de 2017, nas bases de dados e nos portais Medical Literature Analysis and Retrieval System Online (MEDLINE), Biblioteca Virtual em Saúde (BVS), Excerpta Medica database (Embase), Web of Science, Cumulative Index to Nursing and Allied Health Literature (CINAHL) e Scopus Info Site. Os descritores utilizados foram estomia, enfermagem e complicações pós-operatórias. Resultados: Onze artigos responderam à questão norteadora: quais ações de enfermagem podem ser utilizadas na prevenção e tratamento das complicações em estomias intestinais? As complicações mais abordadas foram hérnia paraestomal, estenose, retração da estomia e separação mucocutânea. As ações de enfermagem mais citadas foram demarcação pré-operatória e acompanhamento com enfermeiro especialista no pré- e pós-operatório. Conclusão: A atuação do enfermeiro estomaterapeuta é essencial para a prevenção e tratamento das complicações em estomias intestinais, porém mais estudos de alto impacto são necessários para nortear suas ações.

DESCRITORES: Estomia; Enfermagem; Complicações Pós-operatórias; Estomaterapia.

\footnotetext{
1.Universidade Estadual de Campinas - Faculdade de Enfermagem - Programa de Especialização em Estomaterapia - Campinas/SP - Brasil.

2.Universidade Estadual de Campinas - Hospital de Clínicas - Campinas/SP - Brasil.

3.Hospital Samaritano - São Paulo/SP - Brasil.

4.Prefeitura Municipal de São Paulo - São Paulo/SP- Brasil.

*Autor correspondente: samantha@hc.unicamp.br

Recebido: 28 Ago 2018| Aceito: 28 Mar 2019
} 


\begin{abstract}
Objectives: To identify in the literature the main nursing actions for prevention and treatment of the most frequent complications in intestinal elimination stomies. Methods: Integrative review that followed the phases: identification of the theme and selection of the research question; establishment of criteria for inclusion and exclusion of studies; categorization of studies; evaluation of studies included in the integrative review; interpretation of results; and synthesis of knowledge. The period used was from January 2007 to June 2017, in the databases and in the portals Medical Literature Analysis and Retrieval System Online (MEDLINE), Biblioteca Virtual em Saúde (BVS), Excerpta Medica database (Embase), Web of Science, Cumulative Index to Nursing and Allied Health Literature (CINAHL) and Scopus Info Site. The descriptors used were ostomy, nursing and postoperative complications. Results: Eleven articles answered the guiding question: which nursing actions can be used to prevent and treat complications in intestinal ostomies? The most commonly reported complications were a parastomal hernia, stenosis, retraction of the ostomy and mucocutaneous separation. The most cited nursing actions were preoperative demarcation and follow-up with specialist nurses in the pre- and postoperative periods. Conclusion: The role of the stomatherapist nurse is essential for the prevention and treatment of complications in the intestinal ostomies, but more studies of high impact are necessary to guide their actions.
\end{abstract}

DESCRIPTORS: Ostomy; Nursing; Postoperative Complications; Estomatherapy.

\title{
RESUMEN
}

Objetivo: Levantar en la literatura las principales acciones de enfermería para la prevención y tratamiento de las complicaciones más frecuentes en las estomias intestinales. Método: Revisión integrativa que siguió las fases: identificación del tema y selección de la cuestión de investigación; establecimiento de criterios para la inclusión y la exclusión de estudios; categorización de los estudios; evaluación de los estudios incluidos en la revisión integrativa; interpretación de los resultados y síntesis del conocimiento. El período utilizado fue de enero de 2007 a junio de 2017 en las bases de datos y en los portales Medline, BVS, EMBASE, CINAHL y Scopus. Los descriptores fueron estomia, enfermería y complicaciones postoperatorias. Resultados: Once artículos respondieron a la cuestión orientadora: ¿qué acciones de enfermería pueden ser utilizadas en la prevención y tratamiento de las complicaciones en estomias intestinales? Las complicaciones más abordadas fueron hernia paraestomal, estenosis, retracción de la estomia e separación mucocutánea. Las acciones de enfermería más citadas fueron demarcación preoperatoria y acompañamiento con enfermero especialista en el pre y postoperatorio. Conclusión: La actuación del enfermero estomaterapeuta es esencial para la prevención y tratamiento de las complicaciones en estomias intestinales, pero más estudios de alto impacto son necesarios para orientar sus acciones.

DESCRIPTORES: Ostomía; Enfermeira; Complicaciones posoperativas; Estomaterapia.

\section{INTRODUÇÃO}

O termo estomia pode ser definido, de forma geral, como "construção" cirúrgica de um orifício artificial para fistulização externa de um ducto ou vaso por inserção de um tubo com ou sem sonda de apoio (Descritores de Ciência em Saúde). De forma específica, as estomias intestinais podem ser classificadas como estomias de alimentação ou de eliminação, sendo as mais comuns, nesse último caso, as colostomias e ileostomias ${ }^{1,2}$. As causas que geralmente levam à confecção de estomias de eliminação intestinal são tumores colorretais, doenças inflamatórias intestinais e traumas abdominais ${ }^{3}$. Esse cenário é preocupante, pois, segundo estimativa do Instituto Nacional de Câncer (INCA), 36.360 novos casos de câncer colorretal surgiram no Brasil em 20184; as doenças inflamatórias intestinais apresentam aumento progressivo em sua incidência e prevalência nos países em desenvolvimento, incluindo o Brasil $1^{5}$, e estudos mostram que o trauma abdominal é o tipo mais prevalente entre os politraumas ${ }^{6}$. Independentemente da evolução das técnicas cirúrgicas e da assistência a esse tipo de paciente ao longo dos anos, as complicações das estomias intestinais podem surgir e representam redução da qualidade de vida do indivíduo ${ }^{7} \mathrm{e}$ aumento dos gastos para os serviços de saúde ${ }^{8}$.

As complicações pós-operatórias das estomias são classificadas como imediatas (ocorrem nas primeiras 24 horas após a cirurgia), precoces (entre o $1^{\circ}$ e $7^{\circ}$ dia póscirúrgico) e tardias (após o 7o dia de pós-operatório) 2 .

As complicações imediatas são hemorragia, sangramento, edema e necrose. O edema é uma resposta natural ao procedimento cirúrgico, mas também pode estar associado a uma abertura muito pequena do orifício na parede abdominal e à manipulação excessiva de alça na cirurgia ${ }^{2}$. A necrose da estomia é a morte do tecido devido à redução do fluxo sanguíneo e pode ser superficial ou profunda ${ }^{2}$.

As complicações precoces incluem a separação mucocutânea, na qual ocorre separação da estomia da pele adjacente que pode ser causada por tensão de sutura, 
problemas de cicatrização, infecção ou necrose, e a retração, desaparecimento ou redução importante da estomia na linha da pele do abdome ${ }^{2}$.

Por fim, as complicações tardias incluem estenose, prolapso de alça e hérnia paraestomal. $\mathrm{Na}$ estenose, a estomia encontra-se contraída, impedindo a passagem do efluente, e sua causa pode ser formação de tecido cicatricial em excesso, necrose ou hiperplasia. O prolapso de alça é a exteriorização da alça para além da estomia e pode ocorrer devido à fixação incorreta do intestino à parede do abdome ao menor diâmetro da alça em relação ao orifício da parede abdominal ou aumento da pressão intra-abdominal. A hérnia paraestomal é uma falha na musculatura da parede abdominal que facilita a saída da alça intestinal pelo óstio onde foi realizada a estomia e é causada pela ausência de demarcação pré-operatória correta, fixação inadequada da estomia, fragilidade muscular e/ou pressão intra-abdominal excessiva ${ }^{2}$.

A literatura mostra que pacientes atendidos por enfermeiros estomaterapeutas apresentam complicações menos graves que pacientes que não contam com esse tipo de serviço, necessitando de menos internações cirúrgicas, e menor taxa de internação hospitalar ${ }^{1,9}$.

Estudos demonstram a alta incidência de complicações relacionadas às estomias de eliminação intestinal ${ }^{10,11}$. Nesse cenário, a atuação do enfermeiro, principalmente o especialista estomaterapeuta, torna-se cada vez mais necessária na prevenção e tratamento para redução dessas taxas ${ }^{3}$. Todavia existem poucas publicações que sintetizam as ações de enfermagem para prevenir e tratar essas complicações.

\section{OBJETIVO}

O objetivo deste estudo foi identificar na literatura as principais ações de enfermagem para prevenção e tratamento das complicações mais frequentes nas estomias de eliminação intestinal.

\section{MÉTODOS}

Trata-se de revisão integrativa, método que proporciona a síntese de conhecimento e a incorporação da aplicabilidade de estudos significativos na prática ${ }^{12}$.
A revisão integrativa foi realizada seguindo as fases: identificação do tema e seleção da questão de pesquisa; estabelecimento de critérios para inclusão e exclusão de estudos; categorização dos estudos; avaliação dos estudos incluídos na revisão integrativa; interpretação dos resultados; e síntese do conhecimento dos principais resultados evidenciados na análise dos artigos incluídos ${ }^{12}$.

A questão norteadora foi: identificar e sintetizar na literatura as ações de enfermagem e seus efeitos na prevenção e tratamento das complicações das estomias de eliminação intestinal.

Realizou-se a busca nos portais e bases de dados: Medical Literature Analysis and Retrieval System Online (MEDLINE), Biblioteca Virtual em Saúde (BVS), Excerpta Medica database (Embase), Web of Science, Cumulative Index to Nursing and Allied Health Literature (CINAHL) e Scopus Info Site. Utilizaram-se os descritores em português: estomia, complicações pós-operatórias e enfermagem; em espanhol: ostomía, complicaciones posoperativas e enfermería; e em inglês: ostomy, postoperative complications e nursing. Utilizou-se o operador booleano AND e foram realizadas diferentes combinações, de acordo com a especificidade de cada base de dados.

Os critérios de inclusão para esta revisão foram estudos que tratavam de estomias de eliminação intestinal (colostomias e ileostomias) publicados em inglês, português e espanhol, no período de janeiro de 2007 a junho de 2017, nas bases de dados selecionadas.

Os critérios de exclusão foram artigos que tratavam de outros tipos de estomias que não asde eliminação intestinal (estomias urinárias, estomias de alimentação, como as gastrostomias e jejunostomias, e estomias respiratórias, como as traqueostomias), artigos que tratavam somente da pele periestomia, artigos de revisão, estudos patrocinados por empresas privadas, relatos de caso, artigos de opinião e estudos que não se relacionavam com as ações de enfermagem.

\section{RESULTADOS}

A seleção dos artigos foi feita por dois revisores de forma independente. No primeiro momento, fez-se a leitura dos títulos e resumos da amostra total e, levando-se em consideração os critérios de inclusão 
e exclusão, selecionaram-se 131 artigos dos 569 que retornaram da busca. No segundo momento, fez-se a remoção de 87 artigos duplicados e a amostra foi de 44 artigos (Figura 1). No terceiro momento, fez-se a leitura na íntegra desses 44 artigos, sendo retirados 33 por não atenderem aos critérios de inclusão, chegando à amostra final de 11 artigos.

Para descrição e análise dos estudos, optou-se por utilizar um instrumento de síntese e coleta de dados que contemple os seguintes itens: ano de publicação, objetivo do estudo, tipo de estudo, complicações e ações de enfermagem (Tabela 1).

De 2007 a 2017, a média de publicação foi de um artigo por ano e, em 2014, houve o maior número de publicações sobre o tema (quatro artigos). A maioria dos estudos foi realizada nos Estados Unidos (cinco de 11 artigos) e na Suécia (dois de 11 artigos).

A retração da estomia intestinal foi a complicação mais citada (oito artigos, 72,7\%) seguida por hérnia paraestomal, que apareceu em sete dos 11 artigos (63\%). Estenose, necrose e separação mucocutânea foram abordadas em cinco artigos (45\%). Abcesso, sangramento e fístula foram abordados em três artigos (27\%). Prolapso foi citado em dois artigos (18\%). Obstrução, granuloma, varizes e trauma foram citados em um artigo. A demarcação pré-operatória adequada da estomia foi a ação preventiva mais citada (quatro artigos, 36,3\%). O atendimento pelo enfermeiro estomaterapeuta foi citado como fator importante na prevenção e tratamento das complicações em sete dos 11 artigos (63\%).

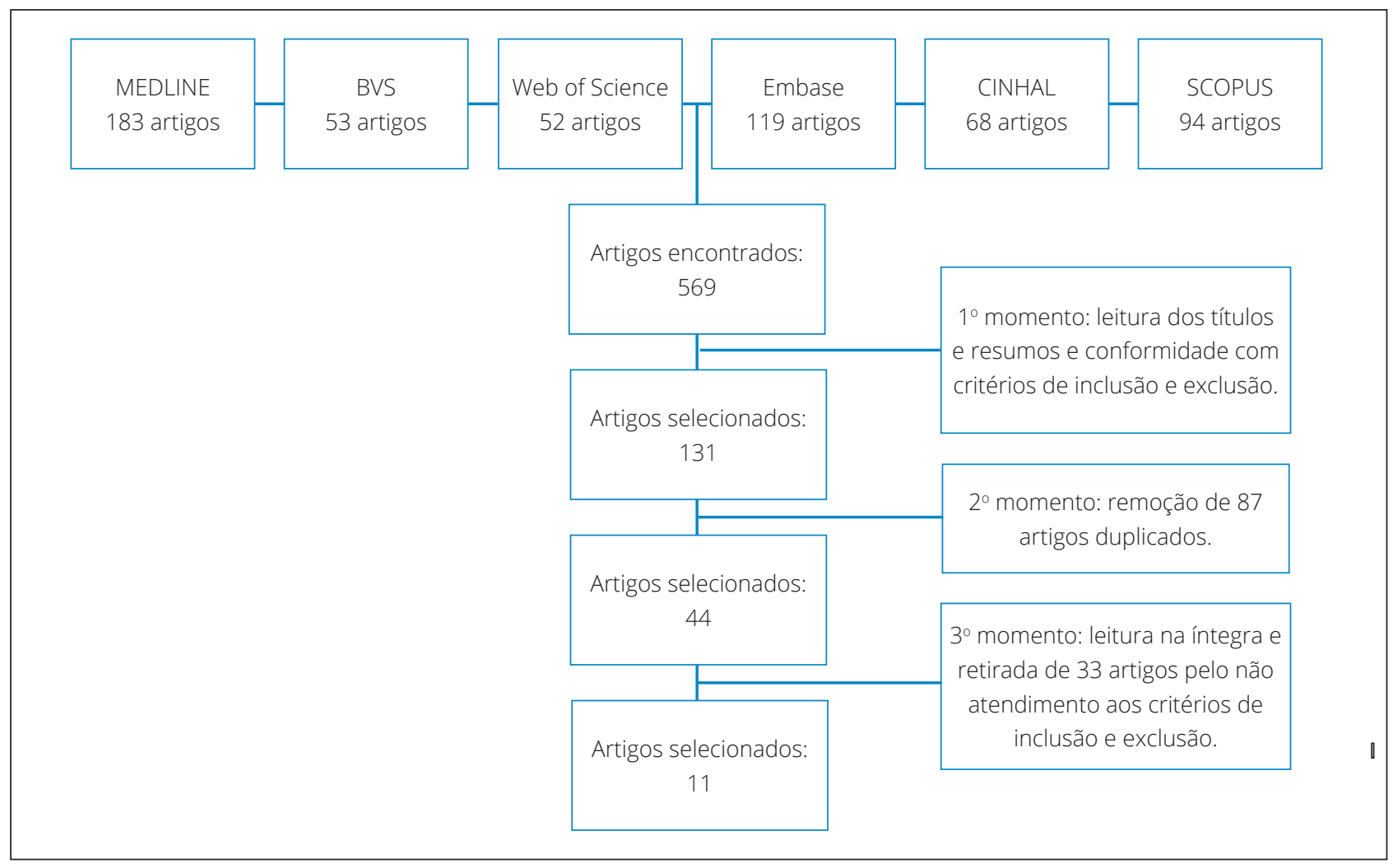

Figura 1. Artigos encontrados avaliados após aplicação dos critérios de inclusão e exclusão.

Tabela 1. Distribuição das publicações segundo ano, objetivo, tipo de estudo, complicações e ações de enfermagem.

\begin{tabular}{|c|c|c|c|c|}
\hline Ano & Objetivo do estudo & Tipo de estudo & Complicações & Ações de enfermagem \\
\hline $2010^{10}$ & $\begin{array}{l}\text { Determinar o tipo e } \\
\text { incidência das complicações } \\
\text { relacionadas a estomias e } \\
\text { identificar fatores associados } \\
\text { em pacientes coreanos }\end{array}$ & $\begin{array}{l}\text { De coorte } \\
\text { retrospectivo }\end{array}$ & 1) Hérnia paraestomal & $\begin{array}{l}\text { 1) Demarcação pré-operatória } \\
\text { correta (dentro músculo reto } \\
\text { abdominal), perda de peso } \\
\text { (obesidade como maior fator de } \\
\text { risco) }\end{array}$ \\
\hline
\end{tabular}


Tabela 1. Continuação...

\begin{tabular}{|c|c|c|c|c|}
\hline $2011^{13}$ & $\begin{array}{l}\text { Desenvolver uma } \\
\text { abordagem padrão para } \\
\text { diagnóstico e tratamento de } \\
\text { complicações de estomias } \\
\text { que otimize a reabilitação de } \\
\text { pacientes e que seja de fácil } \\
\text { uso para não especialistas }\end{array}$ & $\begin{array}{l}\text { Metodológico e } \\
\text { construção de } \\
\text { algoritmo }\end{array}$ & $\begin{array}{l}\text { 1) Sangramento excessivo } \\
\text { 2) Necrose profunda } \\
\text { 3) Retração para a aponeurose } \\
\text { 4) Fístula intestinal alta } \\
\text { 5) Abcesso } \\
\text { 6) Obstrução aguda do intestino } \\
\text { 7) Estenose } \\
\text { 8) Hérnia paraestomal } \\
\text { 9) Prolapso }\end{array}$ & $\begin{array}{l}\text { 1-6) Encaminhamento para cirurgia } \\
\text { de urgência. } \\
\text { 7-9) Encaminhamento para cirurgia } \\
\text { eletiva }\end{array}$ \\
\hline $2012^{14}$ & $\begin{array}{l}\text { Analisar as complicações } \\
\text { que podem ocorrer depois } \\
\text { da confecção de estomia } \\
\text { intestinal }\end{array}$ & $\begin{array}{l}\text { De coorte, } \\
\text { prospectivo }\end{array}$ & $\begin{array}{l}\text { 1) Retração } \\
\text { 2) Hérnia paraestomal } \\
\text { 3) Sangramento }\end{array}$ & $\begin{array}{l}\text { 1-3) Demarcação e orientação } \\
\text { pré-operatória do paciente por } \\
\text { enfermeiro estomaterapeuta }\end{array}$ \\
\hline $2013^{15}$ & $\begin{array}{l}\text { Avaliar prospectivamente a } \\
\text { evolução e incidência das } \\
\text { complicações e descrever } \\
\text { a configuração da estomia } \\
\text { em pacientes submetidos } \\
\text { à cirurgia abdominal de } \\
\text { urgência }\end{array}$ & $\begin{array}{l}\text { De coorte, } \\
\text { prospectivo }\end{array}$ & $\begin{array}{l}\text { 1) Necrose } \\
\text { 2) Separação mucocutânea } \\
\text { 3) Estenose } \\
\text { 4) Prolapso } \\
\text { 5) Hérnia paraestomal } \\
\text { 6) Granuloma }\end{array}$ & $\begin{array}{l}\text { 1-6) Acompanhamento } \\
\text { sistematizado por } 2 \text { anos por } \\
\text { enfermeiro estomaterapeuta (uma } \\
\text { a duas vezes durante internação } \\
\text { hospitalar, } 2 \text { semanas após a alta e } \\
3,6,12 \text { e } 24 \text { meses após a cirurgia) }\end{array}$ \\
\hline $2013^{16}$ & $\begin{array}{l}\text { Avaliar o efeito do } \\
\text { acompanhamento por } \\
\text { telefone pelo enfermeiro } \\
\text { estomaterapeuta na } \\
\text { adaptação pós-alta após } \\
\text { colostomia }\end{array}$ & De intervenção & $\begin{array}{l}\text { 1) Retração } \\
\text { 2) Estenose } \\
\text { 3) Necrose }\end{array}$ & $\begin{array}{l}\text { 1-3) Seguimento sistematizado por } \\
\text { telefone após a alta por enfermeiro } \\
\text { estomaterapeuta; ligações } 3 \text { a } 7 \\
\text { dias após a alta e } 14 \text { a } 20 \text { dias; } \\
\text { uma ligação extra ( } 23 \text { a } 27 \text { dias) foi } \\
\text { feita para os pacientes com mais } \\
\text { dificuldade de adaptação }\end{array}$ \\
\hline $2014^{9}$ & $\begin{array}{l}\text { Avaliar propriedades } \\
\text { pisicométricas de um } \\
\text { instrumento para mensurar a } \\
\text { incidência e a severidade das } \\
\text { complicações pós-operatórias } \\
\text { imediatas das estomias }\end{array}$ & $\begin{array}{c}\text { De coorte } \\
\text { prospectivo }\end{array}$ & $\begin{array}{l}\text { 1) Retração } \\
\text { 2) Separação mucocutânea }\end{array}$ & $\begin{array}{l}\text { 1, 2) Demarcação pré-operatória } \\
\text { por enfermeiro estomaterapeuta }\end{array}$ \\
\hline $2014^{17}$ & $\begin{array}{l}\text { Identificar as intervenções } \\
\text { ideais para as complicações } \\
\text { específicas, com base no } \\
\text { julgamento clínico dos } \\
\text { enfermeiros especialistas }\end{array}$ & $\begin{array}{l}\text { Quantitativo } \\
\text { transversal com } \\
\text { componentes } \\
\text { qualitativos }\end{array}$ & $\begin{array}{l}\text { 1) Hérnia paraestomal } \\
\text { 2) Prolapso } \\
\text { 3) Necrose } \\
\text { 4) Separação mucocutânea } \\
\text { 5) Retração } \\
\text { 6) Estenose } \\
\text { 7) Fístula } \\
\text { 8) Trauma } \\
\text { 9) Varizes }\end{array}$ & $\begin{array}{l}\text { 1) Cinto para apoiar a hérnia. } \\
\text { 2) Equipamento coletor que } \\
\text { acomode todo o comprimento e } \\
\text { largura do estoma. Compressa fria } \\
\text { para reduzir o estoma. } \\
\text { 3) Sistema de bolsa transparente } \\
\text { para visualização direta. } \\
\text { 4) Preenchimento da área separada } \\
\text { com material absorvente (pó ou } \\
\text { pasta de barreira de pele, alginato } \\
\text { de cálcio ou hidrofibra). } \\
\text { 5) Equipamento convexo e cinto. } \\
\text { 6) Aumento da ingestão líquida e } \\
\text { dieta com baixo teor de resíduos. } \\
\text { 7) Equipamento convexo. } \\
\text { 8) Identificar e eliminar o fator } \\
\text { causal. Manejar sangramento com } \\
\text { pressão direta, compressa fria. } \\
\text { 9) Hemostasia com nitrato de prata. }\end{array}$ \\
\hline
\end{tabular}


Tabela 1. Continuação...

\begin{tabular}{|c|c|c|c|c|}
\hline $2014^{3}$ & $\begin{array}{l}\text { Acompanhar e descrever o } \\
\text { efeito da demarcação nas } \\
\text { complicações das estomias e } \\
\text { áreas periestomia }\end{array}$ & $\begin{array}{l}\text { Multicêntrico, } \\
\text { retrospectivo }\end{array}$ & $\begin{array}{l}\text { 1) Separação mucocutânea } \\
\text { 2) Retração }\end{array}$ & $\begin{array}{l}\text { 1, 2) Demarcação pré-operatória } \\
\text { correta, até mesmo nas cirurgias } \\
\text { de urgência; aumento do número } \\
\text { de unidades e enfermeiros } \\
\text { especialistas em estomaterapia }\end{array}$ \\
\hline $2014^{1}$ & $\begin{array}{l}\text { Comparar as complicações } \\
\text { imediatas de pacientes com } \\
\text { estomia que receberam } \\
\text { atenção de enfermeiro } \\
\text { especialista com os que não } \\
\text { receberam }\end{array}$ & $\begin{array}{l}\text { Multicêntrico, } \\
\text { quase } \\
\text { experimental, } \\
\text { prospectivo }\end{array}$ & $\begin{array}{l}\text { 1) Hérnia paraestomal } \\
\text { 2) Hemorragia } \\
\text { 3) Infecção }\end{array}$ & $\begin{array}{l}\text { 1-3) Atendimento por enfermeiro } \\
\text { especialista }\end{array}$ \\
\hline $2016^{18}$ & $\begin{array}{l}\text { Descrição prospectiva da } \\
\text { prevalência de complicações } \\
1 \text { ano após a cirurgia. } \\
\text { Descrever a configuração da } \\
\text { estomia e sua influência nas } \\
\text { complicações }\end{array}$ & $\begin{array}{l}\text { Clínico } \\
\text { prospectivo }\end{array}$ & $\begin{array}{l}\text { 1) Retração } \\
\text { 2) Hérnia paraestomal }\end{array}$ & $\begin{array}{l}\text { 1) Uso de equipamento convexo. } \\
\text { 2) Uso de cinta abdominal. Evitar } \\
\text { exercícios abdominais e carregar } \\
\text { peso nos primeiros } 3 \text { meses após } \\
\text { a cirurgia }\end{array}$ \\
\hline $2016^{19}$ & $\begin{array}{l}\text { Identificar as melhores } \\
\text { intervenções para as } \\
\text { complicações selecionadas, } \\
\text { baseado no julgamento } \\
\text { e expertise dos } \\
\text { enfermeiros especialistas/ } \\
\text { estomaterapeuta }\end{array}$ & $\begin{array}{l}\text { Quantitativo } \\
\text { transversal com } \\
\text { componentes } \\
\text { qualitativos }\end{array}$ & $\begin{array}{l}\text { 1) Prolapso } \\
\text { 2) Necrose } \\
\text { 3) Separação mucocutânea } \\
\text { 4) Retração } \\
\text { 5) Estenose } \\
\text { 6) Fístula estomal }\end{array}$ & $\begin{array}{l}\text { 1) Bolsas adequadas, cintas de } \\
\text { fixação e anéis de silicone para } \\
\text { estabilização. Aplicar compressa fria } \\
\text { em decúbito dorsal por } 10 \text { minutos. } \\
\text { 2) Uso de uma bolsa transparente } \\
\text { de duas peças para melhor } \\
\text { avaliação e indicação do devido } \\
\text { desbridamento. } \\
\text { 3) Preenchimento com material } \\
\text { absorvente e coberto com material } \\
\text { de barreira (anéis moldáveis). } \\
\text { 4) Uso de cinto de apoio, } \\
\text { convexidade e encaminhamento } \\
\text { para revisão cirúrgica. } \\
\text { 5) Dieta de baixo teor de resíduo, } \\
\text { amaciantes de fezes, aumentando a } \\
\text { ingestão de líquidos (se permitido), } \\
\text { dilatação da estomia por profissional } \\
\text { treinado e encaminhamento para } \\
\text { revisão cirúrgica. } \\
\text { 6) Sistema de bolsa incorporando } \\
\text { a estomia e a fístula. Uso de } \\
\text { convexidade }\end{array}$ \\
\hline
\end{tabular}

\section{DISCUSSÃO}

Observou-se que a publicação anual sobre o tema é pequena e que os países com maior nível de desenvolvimento socioeconômico publicaram o maior número de artigos. Apenas um artigo sobre esse tema tinha alto nível de evidência, sendo esse um estudo de intervenção ${ }^{12}$.

Grande parte das ações para prevenção e tratamento das complicações em estomias de eliminação intestinal é de responsabilidade do enfermeiro, seja ele estomaterapeuta ou generalista capacitado. A ação de enfermagem para prevenção de complicações de maior ocorrência em nossa revisão foi a demarcação pré-operatória. A demarcação é considerada o primeiro estágio para adaptação à estomia e permite que essa seja realizada em local adequado, além de ser um momento que possibilita que os indivíduos obtenham informações sobre a confecção do estoma, os cuidados pós-operatórios, os equipamentos e adjuvantes que serão utilizados ${ }^{3}$. Um 
estudo multicêntrico retrospectivo com amostra de 748 pacientes demonstrou que as taxas de complicações foram maiores em pacientes não demarcados no pré-operatório ${ }^{3}$. Esse estudo mostrou que a redução das complicações em estomias intestinais está diretamente relacionada ao maior número de enfermeiros estomaterapeutas e de unidades especializadas em estomaterapia ${ }^{3}$.

O seguimento sistematizado do paciente após a alta hospitalar pelo enfermeiro especialista, ambulatorialmente ou via telefone, principalmente nos dois primeiros anos após a cirurgia, demonstrou ser uma estratégia para redução de complicações ${ }^{18}$. O acompanhamento por telefone pode ser alternativa para serviços que têm alta demanda e demonstra efetividade na adaptação da pessoa à estomia ${ }^{16}$.

A retração da estomia foi a complicação de maior ocorrência em nossa revisão, e a indicação de tratamento para essa condição é o uso de equipamento de flange convexa, de uma ou duas peças. Sua efetividade está em sua capacidade de realizar pressão contra a pele periestomia, aumentando a exteriorização da estomia e permitindo a saída de efluente para dentro do equipamento ${ }^{21}$. A associação do cinto ao aparelho convexo garante sua efetividade e aumenta a segurança do paciente ${ }^{21}$.

A hérnia paraestomal pode ser prevenida com o uso de cinta abdominal, perda de peso (visto que a obesidade é um grande fator de risco para essa complicação), orientação para que o paciente não carregue peso nos primeiros três meses após a cirurgia e a realização de demarcação pré-operatória correta, fazendo com que a estomia fique dentro do músculo reto abdominal ${ }^{10,18}$.

$\mathrm{O}$ aumento da ingesta hídrica, dietas de baixo teor de resíduo, uso medicamentos que tornam o efluente mais amolecido e dilatação da estomia por profissional capacitado são citados na literatura como medidas para controle da estenose da estomia com objetivo de impedir sua obstrução ${ }^{17,19}$. Para condução da necrose, indica-se o uso de bolsa transparente para melhor visualização e evolução, e a separação mucocutânea pode ser tratada com preenchimento com material absorvente (como alginato de cálcio ou hidrofibras) e posterior cobertura com material de barreira (como anéis moldáveis de hidrocoloide $)^{17,19}$.

Todas as ações aqui descritas são desenvolvidas pelos enfermeiros estomaterapeutas diretamente ou indiretamente, o que vai de encontro aos resultados desta revisão. Os estudos analisados mostram que o estomaterapeuta é o profissional-chave na prevenção e resolução das complicações em estomias de eliminação intestinal, atuando em diversas fases da trajetória da pessoa com estomia, promovendo, por meio do cuidado especializado, a independência no seu autocuidado e melhor qualidade de vida ${ }^{3,16}$.

\section{CONCLUSÃO}

Neste estudo, foi possível verificar que a atuação do estomaterapeuta está presente desde a prevenção até o tratamento das complicações de estomias de eliminação intestinal. As ações realizadas pelo enfermeiro estomaterapeuta têm impacto na diminuição das taxas de complicações.

Constatamos que há escassez de publicações com alto impacto e maior rigor metodológico sobre a temática, por isso é necessária a realização de mais estudos a fim de embasar a prática dos enfermeiros, principalmente os especialistas.

\section{CONTRIBUIÇÃO DOS AUTORES}

Conceitualização, Perissotto S, Breder JSC, Zulian LR, Oliveira VX, Silveira NI e Alexandre NMC; Metodologia, Perissotto S, Breder JSC, Zulian LR, Oliveira VX, Silveira NI e Alexandre NMC; Investigação, Perissotto S, Breder JSC, Zulian LR e Oliveira VX; Redação - Primeira versão, Perissotto S, Breder JSC, Zulian LR e Oliveira VX; Redação - Revisão \& Edição, Perissotto S e Breder JSC; Supervisão, Alexandre NMC e Silveira NI.

\section{REFERÊNCIAS}

1. Coca C, Fernández I, Serrano R. Complicaciones tempranas en pacientes portadores de ostomías con y sin atención de enfermería especializada en ostomía. Metas Enferm. 2014;17(1):23-31.
2. Santos VLCG, Cesaretti IUR. Assitência em estomaterapia. Cuidando de pessoas com estomia. 2a ed. São Paulo: Atheneu; 2015. 
3. Baykara ZG, Demir SG, Karadag A, Harputlu D, Kahraman A, Karadag $S$, et al. A multicenter, retrospective study to evaluate the effect of preoperative stoma site marking on stomal and peristomal complications. Ostomy Wound Manage. 2014;60(5):16-26.

4. Instituto Nacional de Câncer. Estimativa de casos novos de câncer colorretal. Rio de Janeiro: INCA; 2018.

5. Ng SC, Shi HY, Hamidi N, Underwood FE, Tang W, Benchimol $\mathrm{El}$, et al. Worldwide incidence and prevalence of inflammatory bowel disease in the 21st century: a systematic review of population-based studies. Lancet. 2017;390(10114):P276978. https://doi.org/10.1016/S0140-6736(17)32448-0

6. Lima SO, Cabral FLD, Pinto Neto AF, Mesquita FNB, Feitosa MFG, Santana VR de. Avaliação epidemiológica das vítimas de trauma abdominal submetidas ao tratamento cirúrgico. Rev Col Bras Cir. 2012;39(4):302-6. https://doi.org/10.1590/ S0100-69912012000400010

7. KaraveliCakirS,OzbayirT.The effect of preoperativestomasite marking on quality of life. Pakistan J Med Sci. 2018;34(1):14953. https://doi.org/10.12669\%2Fpims.341.14108

8. Salvadalena GD. The incidence of stoma and peristomal complications during the first 3 months after ostomy creation. J Wound Ostomy Cont Nurs. 2013;40(4):400-6. https://doi.org/10.1097/WON.0b013e318295a12b

9. Pittman J, Bakas T, Ellett M, Sloan R, Rawl SM. Psychometric evaluation of the ostomy complication severity index. J Wound Ostomy Cont Nurs. 2014;41(2):147-57. https://doi. org/10.1097/WON.0000000000000008

10. Sung YH, Kwon I, Jo S, Park S. Factors affecting ostomyrelated complications in Korea. J Wound Ostomy Cont Nurs. 2010;37(2):166-72. ht $\quad$ htps://doi.org/10.1097/ WON.0b013e3181cf7b76

11. Chilida MSP, Santos AHCA, et al. Complicações mais frequentes em pacientes atendidos em um polo de atendimento ao paciente com estoma no interior do estado de São Paulo. ESTIMA, Braz J Enterostomal Ther. 2007;5(4):31-6.

12. Souza MT de, Silva MD da, Carvalho R de. Integrative review: what is it? How to do it? Einstein (São Paulo). 2010;8(1):1026. https://doi.org/10.1590/s1679-45082010rw1134
13. Kalashnikova I, Achkasov S, Fadeeva S, Vorobiev G. The development and use of algorithms for diagnosing and choosing treatment of ostomy complications: results of a prospective evaluation. Ostomy Wound Manage. 2011;57(1):20-7.

14. Jonkers HAF, Draaisma WA, Roskott AM, van Overbeeke AJ, Broeders IAMJ, Consten ECJ. Early complications after stoma formation: a prospective cohort study in 100 patients with 1-year follow-up. Int J Colorectal Dis. 2012;27(8):1095-9. https://doi.org/10.1007/s00384-012-1413-y

15. Lindholm E, Persson E, Carlsson E, Hallén AM, Fingren J, Berndtsson I. Ostomy-related complications after emergent abdominal surgery a 2-year follow-up study. J Wound Ostomy Cont Nurs. 2013;40(6):603-10. https://doi. org/10.1097/WON.0b013e3182a9a7d9

16. Zhang JE, Wong FKY, You LM, Zheng MC, Li Q, Zhang BY, et al. Effects of enterostomal nurse telephone follow-up on postoperative adjustment of discharged colostomy patients. Cancer Nurs. 2013;36(6):419-28. https://doi.org/10.1097/ NCC.0b013e31826fc8eb

17. Beitz JM, Colwell JC. Stomal and peristomal complications: Prioritizing management approaches in adults. J Wound Ostomy Cont Nurs. 2014;41(5):445-54. https://doi. org/10.1097/WON.0000000000000052

18. Carlsson E, Fingren J, Hallén A-M, Petersén C, Lindholm E. The prevalence of ostomy-related complications 1 year after ostomy surgery: a prospective, descriptive, clinical study. Ostomy Wound Manag. 2016;62(10):34-48.

19. Beitz JM, Colwell JC. Management approaches to stomal and peristomal complications a narrative descriptive study. J Wound Ostomy Cont Nurs. 2016;43(3):263-8. https://doi. org/10.1097/WON.0000000000000215

20. Ursi ES, Galvão CM. Prevenção de lesões de pele no perioperatório: revisão integrativa da literatura. Rev Lat Am Enfermagem. 2006;1(16):124-31. https://doi.org/10.1590/ $\underline{\text { S0104-11692006000100017 }}$

21. Black P. Managing physical postoperative stoma complications. Br J Nurs. 2009;18(17):S4-10. https://doi. org/10.12968/bjon.2009.18.Sup6.44169 